\title{
SOP 01: Clinical Investigations in CESAR
}

\section{Background and Principles}

CESAR Central European Society for Anticancer Drug Research - EWIV (CESAR) is an association of scientists in the area of basic sciences, preclinical oncology and clinical oncology (see also I. Preamble of the Bylaws of CESAR). CESAR is organized in specific working groups dealing with drug development and preclinical pharmacology, Phase I, phase II, and phase III study performance and clinical pharmacology thereby contributing to reach the above defined goals.

Aim of the preclinical and clinical oncologic scientists is to conduct clinical trials in medical oncology, either on behalf of the study group itself or on behalf of external sponsors, like pharmaceutical companies. Such trials have to comply with current guidelines on Good Clinical Practice (GCP) to ensure the acceptability of the data to the scientific community and to drug regulatory authorities. The Note for Guidance on GCP that has been issued by $\mathrm{ICH}$ (International Conference on Harmonisation of Technical Requirements for Registration of Pharmaceuticals for Human Use) is applicable to clinical trials commencing after January 17, 1997 [1]. Because of convenience, these GCP guidelines are referred to as 'ICH-GCP' within this SOP series.

The SOPs of CESAR define or address exclusively requirements for clinical activities but do not apply basic scientific and preclinical activities, that are covered by $\mathrm{CE}$ SAR-AWO regulations.

According to ICH-GCP, the sponsor of a clinical trial has to verify the suitability of each participating investigator and institution [1; section 5.6.1]. It is the aim of CESAR to shorten the lead time for the initiation of a particular clinical trial by applying and documenting appropriate procedures before granting a group membership. The selection criteria for becoming a member in a clinical working group (phase I, phase II, phase III) cover both the medical qualification and experience of the medical oncologist as well as the organization and equipment of the institution to which he/she is affiliated.
A cumulative data base on the number of patients with key indications that working group members have treated will support the identification of institutions with adequate recruitment rates for a new clinical trial.

\section{Becoming a Member of a (the) Working Group(s) of CESAR}

Acceptance for membership in the working group(s) of CESAR will be based on both the scientific qualification of an applicant and on the fulfillment of the technical and organizational prerequisites for performing phase I or phase II trials at the applicant's institution. Thus, membership will neither be sustained automatically if a member changes his/her institutional affiliation nor be transferred to a successor at the institution he/she is leaving.

\section{Selection Criteria for Becoming a Clinical Investigator of CESAR}

It is the aim of CESAR to ensure that each clinical investigator of each CESAR working group fulfills the basic requirements for being an investigator according to $\mathrm{ICH}$ GCP. Compliance with study-specific eligibility criteria will be required to participate in a particular study of CESAR.

- Coordinating Investigator (CI)/Principal Investigator (PI)

- Licensed as physician with at least 2 years of experience in clinical trials (as evidenced by a current curriculum vitae including scientific training and list of publications) (see Attachment 1 for corresponding declaration).

- $\quad C I$ is the only investigator determined by CESAR to guide a specific study on behalf of the society. He will be identified by the chairman of the respective working group.

- $P I$ is each investigator responsible for one center regarding the participation in a specific study on behalf of CESAR who reports to the CI with regard to a distinct study.

\section{KARGER}

Fax +497614520714

E-mail Information@Karger.de

www.karger.com
(C) 2003 S. Karger GmbH, Freiburg 


\section{- Investigator}

- Long-term affiliation to the corresponding institution (affiliation to an institution by a short-term contract, e.g., for less than 2 years, will not be acceptable for a CI/PI of CESAR, as maintenance of an adequate infrastructure for clinical trials meeting the standards of CESAR may not be assured under such circumstances).

- Commitment to participate in quality assurance measures as required by ICH-GCP.

- Organizational Prerequisites at the Study Center (see Attachment 2)

- Identification and documented composition of the relevant local Ethics Committee (policy regarding votes from an outside CI must be known).

- Adequate staff:

experienced medical and nursing staff for the management of study patients,

adequately trained and experienced medical doctor who will be in charge of the study during transient absence of the Full Member (principal investigator),

documentation (as clinical trial protocols and case record forms (CRFs) are often written in English and CRF entries may be required in English, persons assigned the responsibility for documentation should be adequately skilled in English),

drug accountability (storage, distribution and return of trial medication).

- Facilities required for trials, such as:

local access to imaging techniques that are commonly required to measure target lesions (e.g. radiography, CT scan, MRI),

access to hospital beds for inpatient observation,

direct access to an intensive care unit/resuscitation equipment (depending on type of study),

quality assurance of diagnostic procedures (e.g. by participation in quality assurance programs) (see Attachment 3 regarding clinical laboratory),

access to a clinical pharmacological laboratory for test drug or metabolite assays (depending on the analytes to be detected, the cooperation with an external institution may be necessary; for such cases adequate freezer capacity and access to dry ice for shipment of biological specimens must be available).

- Adequate facilities for storage of study materials such as study files and trial medication in a secure, limited access area.

Adequate facilities for later archiving of the 'essential documents' (ICH-GCP) after completion of the study (see SOP 11).

- Equipment for electronic messages and information transfer.
- Availability of patients who may be eligible for clinical trials

- The type of patients that an investigator's institution commonly sees is a critical issue in assessing the suitability of that investigator and institution for participating in a particular clinical trial. Therefore, an applicant for a membership in one or several working groups of CESAR is expected to present an overview of the kind of patients that have been treated in the applicant's institution during the last year (see attachment 'Declaration on the Investigator's Institution'). The focus should be on the number of patients who would meet common selection criteria for phase I or phase II trials and on how many patients actually entered clinical trials. Such information will serve as a basis for reliably estimating accrual rates for trials of the working groups.

- After nomination as a member of a working group, the Declarations may be used to assess an individual study center's suitability to participate in a particular trial project (for details see SOP 6). For this reason, all members of the working group have to update this checklist at regular intervals and submit notes on relevant changes at their institution.

The checklist can serve to facilitate the decisionmaking process when selecting the most suitable institutions for trials involving a limited number of centers. A participating center can be excluded from an ongoing study or from the working group in case of considerable overestimation of its own capacities or severe deficiencies in study documentation (for details see Sections 1.2.6 and 1.2.7).

- The above checklist is supplemented by an annual report of the Data Center on the working group at the biometry of CESAR at the Deutsches Krebsforschungszentrum (German Cancer Research Center) on each trial center's performance level during the preceding year:

- Patient accrual by indications

- Protocol violations

- Patients lost to follow-up

- Delays in the submission of data collection forms.

\section{References}

1 ICH-GCP: EMEA. Note for Guidance on Good Clinical Practice (CPMP/ICH/135/95).

\section{Appendices}

Attachment 1: Investigator's Declaration

Attachment 2: Declaration on the Investigator's Institution Attachment 3: Validated Laboratory Normal Ranges 

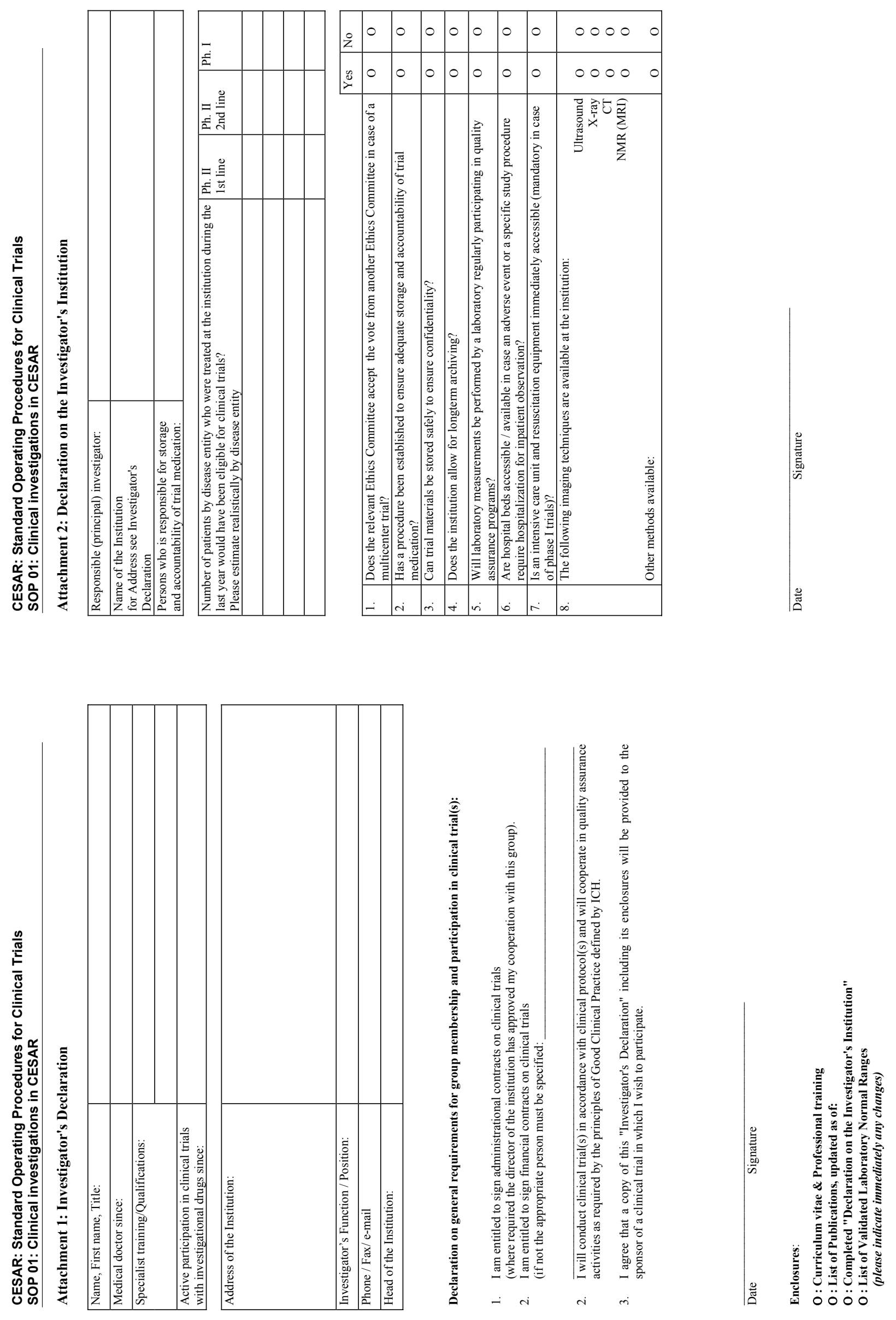

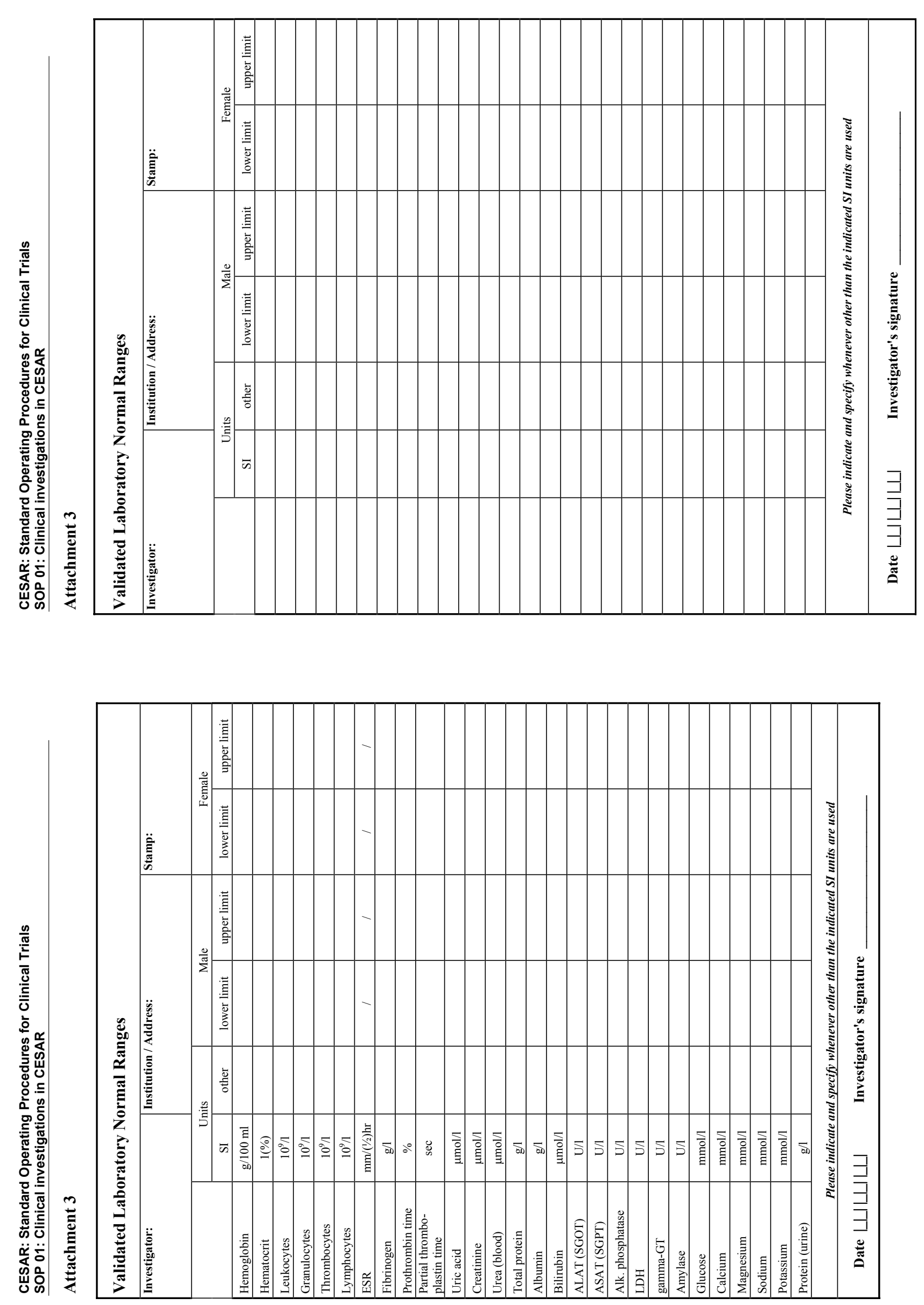